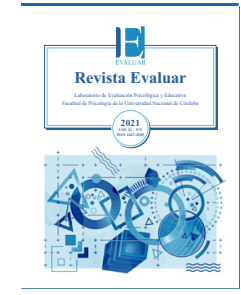

\title{
Validez de contenido del Cuestionario de Ciberagresión
}

\section{Content validity of the Cyber Aggression Questionnaire}

\author{
Silvana Best * ${ }^{1}$, Nancy Ré ${ }^{2}$, Lucie Corcoran ${ }^{3}$, Conor Mc Guckin ${ }^{4}$ \\ 1 - Universidad de Ciencias Empresariales y Sociales, Rafaela, Argentina. \\ 2 - Universidad de Ciencias Empresariales y Sociales, Ciudad Autónoma de Buenos Aires, Argentina. \\ 3 - Dublin Business School, Dublin 2, Irlanda. \\ 4 - Trinity College Dublin, Dublin 2, Irlanda.
}

Introducción

Método

Resultados

Discusión

Referencias

Recibido: 23/10/2020 Revisado: 22/12/2020 Aceptado: 11/01/2021

\section{Resumen}

El propósito de este trabajo es presentar el proceso de validación de contenido del Cuestionario de Ciberagresión diseñado en Irlanda por Corcoran y Mc Guckin (2014). En este estudio instrumental participaron 15 jueces locales, con experticia y trayectoria en la temática, quienes ponderaron cuantitativa y cualitativamente el cuestionario. Se han tomado en cuenta todas las aportaciones realizadas en el análisis cualitativo. Los datos cuantitativos se sistematizaron utilizando el coeficiente $\mathrm{V}$ de Aiken complementado con el uso de intervalos de confianza. Los resultados indican un amplio grado de acuerdo entre los jueces, en la medida en que presentan intervalos de confianza superiores a .50. Por todo ello, se concluye que el Cuestionario de Ciberagresión es una herramienta adecuada para medir dicho constructo en adolescentes escolarizados de Argentina. El presente estudio ofrece el primer instrumento en español válido para medir dicho fenómeno.

Palabras clave: ciberagresión, evaluación psicológica, validez de contenido, juicio de expertos, coeficiente V de Aiken

\section{Abstract}

The purpose of this work is to present the content validation process of the Cyberbullying Questionnaire designed in Ireland by Corcoran and Mc Guckin (2014). In this instrumental study, 15 judges with expertise and experience in the subject participated, who quantitatively and qualitatively weighted the questionnaire. Regarding the qualitative analysis, all contributions were taken into account. The quantitative data were systematized using Aiken's V coefficient supplemented with the use of confidence intervals. The results indicate a wide degree of agreement among the judges, as they present confidence intervals greater than .50. Therefore, it is concluded that the Cyber-aggression Questionnaire is an adequate tool to measure this construct in school-going adolescents in Argentina. This study offers the first valid instrument in Spanish to measure this phenomenon.

Keywords: cyber-aggression, psychological assessment, content validity, expert judgment, Aiken validity coefficient

*Correspondencia a: Silvana Best. 25 de Mayo 906, Rafaela, Santa Fe, Argentina. Tel. +54 3492 434198. E-mail: sbest@campus.uces.edu.ar Cómo citar este artículo: Best, S., Ré, N., Corcoran, L., \& Mc Guckin, C. (2021). Validez de contenido del Cuestionario de Ciberagresión. Revista Evaluar, 21(2), 1-16. Recuperado de https://revistas.unc.edu.ar/index.php/revaluar Participaron en la edición de este artículo: Julian Narvaja, Stefano Macri, Juan Cruz Balverdi, Alicia Molinari, Florencia Ruiz, Ricardo Hernández. 


\section{Introducción}

El escenario de la comunicación se ha modificado vertiginosamente en los últimos tiempos debido a la proliferación de diversas pantallas: desde la aparición del televisor hasta las más recientes de los videojuegos, la computadora, internet, y el teléfono celular (Bringué-Sala, Sádaba-Chalezquer, \& Artopoulos, 2014). Niños, adolescentes y jóvenes constituyen el segmento de edad que más rápida y precozmente incorpora en sus vidas un universo de posibilidades a través de herramientas tecnológicas con las que se relacionan de modo habitual. La popularización de los teléfonos inteligentes ha producido una fusión creciente de la telefonía celular e internet, convirtiendo a ambas en medios esenciales para la socialización de los adolescentes (Álvarez-García, Dobarro, \& Núñez, 2015). En la actualidad, el uso de teléfonos móviles entre los adolescentes y jóvenes argentinos es prácticamente universal: el $96.8 \%$ afirma tener este aparato y utilizarlo para comunicarse con otros (Bringué-Sala et al., 2014; Secretaría de Evaluación Educativa, 2017). Es decir, los usos comunicativos de la tecnología destacan sobre las demás posibilidades de uso de estas.

En este sentido, si bien tanto el teléfono móvil como internet son herramientas útiles para establecer y mantener relaciones sociales de diversas características, no siempre se hace un uso adecuado de ellas, lo que da lugar a ciertos riesgos potenciales. Uno de ellos es el empleo de estos medios para molestar, ofender o perjudicar de manera deliberada a otras personas. El término cyberbullying fue inicialmente una denominación conveniente para designar las conductas abusivas y agresivas perpetradas a través de teléfonos móviles e internet (Corcoran, Mc Guckin, \& Prentice, 2015). Fue Olweus (2012) quien advirtió acerca de la dificultad que acarrearía utilizar el mis- mo vocablo para apresar fenómenos que pueden presentar características diferentes: el bullying implica una modalidad de relación específica, por lo cual no sería conveniente utilizarlo para denominar cualquier acto agresivo o negativo (Hunter, Boyle, \& Warden, 2004; Olweus, 2010). Como señala Garaigordobil (2011), existe un problema con la definición de cyberbullying, por ser un término complejo y, por lo tanto, difícil de operacionalizar conforme con los criterios consensuados por la comunidad científica para el bullying tradicional: intención de dañar, repetición de las conductas y desequilibrio de poder.

Algunos investigadores que han estudiado el cyberbullying han puesto el énfasis en el carácter específico de este fenómeno, diferenciándolo de la ciberagresión en general (Zych, Ortega-Ruíz, \& Marín-López, 2016). Sin embargo, otros, como Bauman, Underwood y Card (2013), sugieren, por el contrario, que los estudios sobre el cyberbullying no son lo suficientemente específicos y, por lo tanto, el concepto que actualmente está en estudio es el de ciberagresión.

Dorothy Grigg (2010), con su trabajo "Cyber-Aggression: Definition and Concept of Cyberbullying", inauguró esta nueva línea de investigación que coloca el foco de atención en la amplia gama de conductas negativas que pueden ocurrir en el ciberespacio. La autora define la ciberagresión como "daño intencional efectuado a través de medios electrónicos a una persona o grupo de personas independientemente de su edad, quienes perciben esos actos como ofensivos, despectivos, perjudiciales o no deseados" (p. 152). Retomando dicha conceptualización, Corcoran et al. (2015) definen a la ciberagresión como cualquier conducta realizada a través de las nuevas tecnologías de la información y la comunicación que tiene la intención de dañar a una o varias personas, quienes desean evitar esta situación. La intención de dañar deberá ser juzgada sobre la base de cómo 
una persona razonable percibe dicha conducta. $\mathrm{Si}$ bien no existe aún consenso respecto de si la ciberagresión es una conducta que puede ser identificada como acoso en los entornos cibernéticos, lo valioso del constructo reside en su carácter general, que posibilita explorar el conjunto de las conductas negativas online. En el estudio realizado en Irlanda por Corcoran (2013) con una muestra de 2747 adolescentes irlandeses escolarizados, donde se analizó la prevalencia y características de la ciberagresión, el cyberbullying y el bullying, se observó que las experiencias de ciberagresión presentaban una tasa mayor que las de cyberbullying. En el caso de la ciberagresión sufrida, el $57 \%$ de los encuestados declaró haberla padecido en los últimos tres meses mientras que respecto de la cibervictimización solo el 14\% expresó haberla vivenciado. Es decir, resulta factible que la ciberagresión constituya uno de los riesgos online más extendidos entre la población adolescente.

Los entornos a través de los cuales se desarrollan estos comportamientos les otorgan determinadas características e impacto en el desarrollo psicosocial de los adolescentes, que los hacen especialmente problemáticos y dignos de atención (Álvarez-García, Barreiro-Collazo, \& Nuñez, 2017). Los entornos cibernéticos favorecen el anonimato por parte del agresor y, con ello, la desinhibición en su conducta. El agresor suele no presenciar las consecuencias sobre quien sufre la agresión, lo que dificulta la empatía. Sumado a ello, las agresiones pueden ocurrir en cualquier momento y lugar, lo que complica la supervisión y control por parte de los adultos. Además, el contenido dañino puede ser enviado a mucha gente en muy poco tiempo, con la consecuente multiplicación del daño (Hinduja \& Patchin, 2015). Entre los efectos negativos de estas conductas, se ha observado que sufrir agresiones online se asocia a un aumento de problemas emocionales, especialmente aquellos relacionados con sintomatolo- gía depresiva, que en adolescentes puede afectar negativamente la concentración y el rendimiento académico (Kowalski, Giumetti, Schroeder, \& Lattanner, 2014). Quienes perpetran agresiones en línea pueden ver incentivado este comportamiento, favoreciendo su generalización a otros ámbitos y situaciones (Álvarez-García et al., 2017; Yahner, Dank, Zweig, \& Lachman, 2015). La ciberagresión, cuando es detectada, puede conllevar importantes consecuencias legales para quienes la ejercen o posibilitan (Paul, Smith, \& Blumberg, 2012).

Con respecto a la prevalencia de las conductas agresivas online, no hemos hallado estudios de este tipo desarrollados en Argentina. El Ministerio de Educación incluyó en las evaluaciones Aprender para estudiantes primarios y secundarios un breve apartado sobre su percepción de las distintas expresiones de violencia escolar, entre ellas, las cibernéticas. Los datos de 2017 respecto de estudiantes del último año de educación secundaria revelaron que un 54\% de la población adolescente evaluada describía agresiones a través de las redes sociales (Secretaria de Evaluación Educativa, 2017). El escaso interés de la investigación en este campo es un hecho constatable también en otros países de América Latina (Lacunza, Contini, Caballero, \& Mejail, 2019; Smith \& Berkkun, 2017; Zych, Ortega-Ruiz, \& del Rey, 2015). La situación planteada abona la hipótesis de que una mejor aproximación a la medición del comportamiento agresivo en entornos cibernéticos apoyaría los esfuerzos destinados a reducir la incidencia de este tipo de comportamientos dañinos (Corcoran et al., 2015). Un estudio realizado por el Fondo de las Naciones Unidas para la Infancia (Paolini \& Ravalli, 2016) señala el valor de investigar el vínculo de los adolescentes con la tecnología a fin de generar evidencia que posibilite la toma de decisiones sobre políticas referidas a ciudadanía digital y alfabetización digital (Lacunza et al., 
2019). En los últimos años se ha acrecentado el interés por la adaptación de instrumentos de medición en todos los ámbitos de la evaluación psicológica, reflejo de un medio social marcado por los contactos entre culturas e idiomas diferentes. Sin embargo, se observa en nuestro país una gran escasez de estudios empíricos respecto de la temática objeto del presente estudio, que obedecería en parte a la falta de instrumentos validados en él. Ello afecta la práctica de los actores involucrados en diversos campos profesionales, puesto que los tests y cuestionarios asisten diariamente en los ámbitos educativo, social, jurídico y clínico, en la toma de decisiones (Muñiz \& Hambleton, 1996). Resulta, por lo tanto, imperioso disponer de instrumentos de evaluación validados en población argentina, que permitan comprender el fenómeno, detectar posibles casos, diseñar intervenciones y evaluar la eficacia de las mismas.

El Cuestionario de Ciberagresión de Corcoran y Mc Guckin (2014) constituye un autoinforme diseñado para medir, en sujetos de 12 a 18 años de edad, con qué frecuencia el informante es víctima o perpetrador de agresiones a través del teléfono móvil e internet, además del tipo de agresión que sufre o comete. Sus áreas de aplicación son la investigación y el screening. Este tipo de instrumentos tiene la ventaja de poder evaluar a una gran cantidad de personas en un lapso corto de tiempo, lo cual resulta muy eficaz no solo como medida de screening sino, sobre todo, en estudios epidemiológicos. No se han hallado otros antecedentes de cuestionarios que evalúen de forma específica el constructo ciberagresión en sus dos modalidades: ciberagresión sufrida y ciberagresión perpetrada. Los cuestionarios desarrollados hasta el momento se abocan a medir el cyberbullying, considerando a la ciberagresión como una dimensión de este junto con la cibervictimización. Entre los cuestionarios no específicos de ciberagresión hallados, algunos han sido diseñados para evaluar con qué frecuencia el informante es agresor o víctima de acoso a través del teléfono móvil o internet. Este es el caso del European Cyberbullying Intervention Project Questionnaire (ECIPQ) de Brighi et al. (2012), el Cyberbullying Questionnaire (CBQ) de Gámez-Guadix, Villa-George y Calvete (2014) y la Cyberbullying Scale (CS) de Menesini, Nocentini y Calussi (2011). Cabe señalar que el European Cyberbullying Intervention Project Questionnaire (ECIPQ) fue adaptado y validado en población colombiana por Herrera-López, Casas, Romera, Ortega-Ruiz y del Rey (2017). Otros han sido diseñados para evaluar con qué frecuencia el informante es agresor, víctima u observador de violencia a través del teléfono móvil e internet. Tal es el caso del test Cyberbullying (Garaigordobil, 2013), validado en población mexicana escolarizada por Laca-Arocena, Pérez-Verduzco, Luna-Bernal, Carrillo-Ramírez y Garaigordobil (2020); o de la Subescala de Agresión Virtual en Escolares de Jiménez, Castillo y Cisternas (2012). Por esta razón, el Cuestionario de Ciberagresión de Corcoran y Mc Guckin (2014) constituye una importante fuente de información para delimitar de manera empírica los indicadores que conforman el constructo y analizar la posible existencia de tipos diferenciados. Fue confeccionado a partir del cuestionario denominado "Copying with Ciberbullying Questionnaire (CWCBQ)", diseñado por Fabio Sticca et al. (2015) en el marco del Estudio netTEEN (Suiza) y financiado por la Swiss National Science Foundation (SNF No. 100014_130193/1). El CWCBQ fue utilizado y validado en Suiza $(\mathrm{N}=803)$, Italia $(\mathrm{N}=755)$ e Irlanda $(\mathrm{N}=2412)$ con muestras de adolescentes de entre 12 y 18 años. Utilizando el Cuestionario CWCBQ (Copying with Ciberbullying Questionnaire) de Sticca como antecedente, Corcoran y Mc Guckin (2014) lo adaptaron para población irlandesa en estrecha colaboración con el equipo 
suizo. Sin embargo, el Cuestionario de Ciberagresión permite medir un constructo diferente al del Cuestionario de Sticca y colaboradores (Machmutow, Perren, Sticca, \& Alsaker, 2012; Sticca et al., 2015), ya que se centra en la ciberagresión.

La investigación efectuada en Irlanda con el Cuestionario de Ciberagresión de Corcoran y Mc Guckin (2014) con una muestra de 2474 estudiantes irlandeses comprendidos en un rango de edad de entre 12 y 18 años, arrojó un coeficiente alfa de Chronbach de .82 para la Escala Ciberagredido y un .78 para la Escala Ciberagresor. Estos resultados alientan su validación y desarrollo en nuevos estudios con muestras poblacionales de otras geografías. No obstante, debe señalarse como limitación que los autores del Cuestionario de Ciberagresión no han realizado ningún otro tipo de estudio acerca de la validez de este. Hasta el momento, no se han encontrado investigaciones en las que se haya utilizado el citado cuestionario y se haya realizado un estudio acabado de su validez ni de sus propiedades psicométricas, como el que aquí se propone. La fase de adaptación transcultural del cuestionario implicó el desarrollo de cinco acciones (Best, Ré, Mc Guckin, Corcoran, \& Casasnovas, 2017):

1. Traducción inicial del inglés al español a cargo del equipo de investigación.

2. Análisis de la equivalencia lingüística y semántica del término cyberaggression y de las palabras anglosajonas asociadas cyberbullying y bullying, con vocablos del idioma español. Para esta fase, se desarrollaron cuatro grupos focales con adolescentes escolarizados de 13 a 18 años de CABA y Rafaela. Asimismo, se administró un cuestionario complementario a una muestra por conveniencia de estudiantes de la misma franja etaria de ambas ciudades.

3. Traducción y retraducción inversa a cargo de dos traductores matriculados. La versión en inglés producto de la traducción inversa fue evalua- da por los autores del cuestionario.

4. Elaboración de la versión argentina preliminar del Cuestionario y análisis de la validez de apariencia de esta a través de la aplicación de una prueba piloto.

5. Juicio de expertos.

El objetivo del presente trabajo es analizar la validez de contenido del Cuestionario de Ciberagresión de Corcoran y Mc Guckin (2014) por medio de la valoración de jueces expertos desde el enfoque de los intervalos de confianza. El propósito de este análisis es encontrar indicadores rigurosos de la representatividad de los ítems de cada escala y realizar los cambios necesarios a nivel lingüístico para hacer viable su uso en nuestro medio, dado que, en la actualidad, tal como se ha mencionado, no se cuenta con instrumentos adaptados y validados con población argentina que posibiliten el estudio de la ciberagresión.

\section{Metodología \\ Tipo de estudio}

Se trata de un estudio instrumental, destinado a la adaptación y estudio de las propiedades psicométricas de un cuestionario (Montero \& León, 2002).

\section{Participantes}

Tanto la selección como la determinación del número de los expertos se ha realizado conforme a criterios establecidos por la comunidad científica. Para Cabero-Almenara y Llorente-Cejudo (2013), si se tiene en cuenta que "el concepto de experto es bastante polisémico", su correcta aplicación depende de los criterios de selección y del número adecuado de los mismos.

En la presente investigación participaron 15 
jueces locales expertos en la temática. Los jueces se seleccionaron de acuerdo con los criterios enunciados por Skjong y Wentworth (2001): experticia (grados, investigaciones, publicaciones y experiencia), reputación, disponibilidad para participar e imparcialidad. Se atendió además al criterio formulado por Escobar-Pérez y Cuervo-Martínez (2008) para los casos de traducciones y adaptaciones de pruebas: se requiere por lo menos un experto en lingüística. Se conformó un panel de expertos con las siguientes características: el 90\% de los jueces seleccionados posee título de doctor; el 10\% posee título de máster y/o especialista; cuatro son expertos en medición y evaluación psicológica; uno es experto en lingüística; cinco son expertos en el campo de problemáticas que releva el Cuestionario y cinco poseen experticia en Psicología de la Adolescencia y Psicología Educacional.

\section{Procedimiento}

La estimación de la validez de contenido del Cuestionario de Ciberagresión de Corcoran y Mc Guckin (2014) se realizó, tal como se ha mencionado, utilizando la técnica del juicio de expertos. Tal como afirman Escobar-Pérez y Cuervo-Martínez (2008), en el caso de la psicología dicho juicio se ha convertido en la estrategia principal para la estimación de la validez de contenido. El juicio de expertos se define como una opinión informada de personas con trayectoria en el tema, que son reconocidas por otros como expertos cualificados en este, y que pueden dar información, evidencia, juicios y valoraciones (McGartland-Rubio, Berg-Weger, Tebb, Lee, \& Rauch, 2003).

Se realizó un contacto inicial con los expertos del ámbito local a través de correo electrónico, exponiéndoles el objetivo de la validación, así como una explicación acerca de la tarea que implicaba la valoración de la escala por parte de ellos mediante un formato de cuestionario online.

Se elaboró una planilla de calificación online para los expertos respetando los lineamientos metodológicos sugeridos por Escobar-Pérez y Cuervo-Martínez (2008). La misma brinda al juez información acerca del objetivo del juicio de expertos, características del Cuestionario de Ciberagresión, definición conceptual y operativa del constructo e indicaciones para su completamiento. La aplicación utilizada fue Google Docs.

Cada juez realizó una valoración de cada ítem del Cuestionario, atendiendo a tres categorías: a) Pertinencia: ¿El ítem apunta a obtener la información que se está buscando?; b) Claridad: ¿El ítem es claro y no admite más de una interpretación posible?, y c) Redacción: ¿El ítem está correctamente formulado? ¿El lenguaje es apropiado?

Al cierre, se consignó un apartado de observaciones que quedó abierto para incluir las sugerencias que los jueces creyeran pertinentes, puesto que el análisis cualitativo se considera un requisito indispensable en el desarrollo de un instrumento (Bulger \& Housner, 2007; McGartland-Rubio et al., 2003). Las aportaciones realizadas por los jueces en este apartado fueron cuidadosamente discutidas y analizadas por los investigadores.

Los resultados cuantitativos se sistematizaron utilizando para ello el coeficiente de validez V de Aiken (Aiken, 1980; Escurra-Mayaute, 1988) para la validación por criterio de jueces, complementada con el uso de intervalos de confianza, requerido en la actualidad por estándares internacionales (Fidler, 2002) y desarrollado por Penfield y Giacobbi (2004).

El coeficiente de validez V se calculó sobre las valoraciones del conjunto de jueces respecto de cada ítem. Las valoraciones asignadas fueron politómicas, con una escala de valores de uno a 
cinco. El coeficiente $\mathrm{V}$ de Aiken indica el grado de acuerdo entre los jueces respecto a la adecuación del ítem al criterio evaluado. Un valor $\mathrm{V}$ mayor a .7 puede considerarse indicador de validez de contenido, valores menores a .7 indican la necesidad de reconsiderar el ítem y/o reformularlo. Sin embargo, dado que el coeficiente $\mathrm{V}$ podría estar influido por error muestral, se recomienda utilizar este coeficiente considerando simultáneamente los límites superior e inferior del intervalo de confianza, que indican los máximos y mínimos valores que podría asumir el parámetro. Se ha sugerido que valores del límite inferior superiores a .5 señalan que el ítem es representativo, mientras que valores inferiores requieren considerar con mayor precaución la validez del mismo (Cicchetti, 1994; Merino-Soto, 2018; Merino-Soto \& Livia-Segovia, 2009).

\section{Instrumento}

Se utilizó el Cuestionario de Ciberagresión diseñado y validado en Irlanda por Corcoran y Mc Guckin (2014), autoinforme diseñado para medir, en sujetos de 12 a 18 años de edad, con qué frecuencia el informante es víctima o perpetrador de agresiones a través del teléfono móvil e internet, además del tipo de agresión que sufre o comete.

El constructo está compuesto por dos dimensiones que son exploradas respectivamente en cada subescala: agresión sufrida (Escala Ciberagredido) y agresión perpetrada (Escala Ciberagresor). En la adaptación argentina del Cuestionario, se las denomina Escala Ciberagredido y Escala Ciberagresor, para evitar confundir la ciberagresión sufrida con cibervictimización.

Tal como se muestra en las Tablas 1 y 2 , en cada escala se exploran diversos tipos de ciberagresión clasificados según las tipologías de Nocentini et al. (2010) y Langos (2012).
El informante valora la frecuencia con que sufrió o perpetró cada tipo de conducta agresiva online durante los últimos tres meses mediante una escala de respuesta tipo Likert. El ítem 9 de ambas escalas constituye un ítem de respuesta abierta donde se pregunta a cada encuestado si ha experimentado cualquier otra forma de agresión cibernética no mencionada en los ítems anteriores. También se le solicita que indique la frecuencia de estas situaciones utilizando la misma escala tipo Likert de los anteriores ítems. De este modo, se reconoce e incluye el rasgo dinámico y cambiante del ciberespacio que, por ejemplo, al desarrollar nuevos dispositivos o aplicaciones posibilita la emergencia de conductas negativas aún no estudiadas.

\section{Resultados \\ Escala Ciberagredido}

La Escala Ciberagredido está conformada por los siguientes ítems:

1. ¿Alguien te envió mensajes desagradables o amenazantes a través del teléfono celular o internet?

2. ¿Alguien te envió imágenes o videos ofensivos o amenazantes a través del teléfono celular o internet?

3. ¿Alguien envió mensajes escritos ofensivos o divulgó rumores sobre vos a través del teléfono celular o internet?

4. ¿Alguien envió imágenes o videos ofensivos o amenazantes sobre vos a través del teléfono celular o internet?

5. ¿Alguien publicó mensajes escritos ofensivos o divulgó rumores sobre vos a través del teléfono celular o internet?

6. ¿Alguien publicó imágenes o videos ofensivos o amenazantes sobre vos a través del teléfono celular o internet? 
7. ¿Alguien usó tu nombre de usuario y contraseña e hizo cosas ofensivas o amenazantes?

8. ¿Alguien te impidió participar, sin motivo, de alguna actividad en línea (por ej. de un juego o grupo creado en una red social)?

9. ¿Alguien te agredió de alguna otra manera usando Internet o el teléfono celular? Si la respuesta es sí, describí lo que pasó y marcá la casilla correspondiente.

Las tablas a continuación dan cuenta de las $\mathrm{V}$ de Aiken y los intervalos de confianza al 90\% de los ítems que componen la Escala Ciberagredido.

En la Tabla 3 se incluyen los valores medios obtenidos en cada ítem para cada uno de los crite- rios evaluados (pertinencia, claridad, redacción) $\mathrm{y}$, por último, los puntajes medios obtenidos por cada ítem. Junto a las medias se adjunta el coeficiente $\mathrm{V}$ de Aiken que indica el grado de acuerdo entre los jueces respecto a la adecuación del ítem al criterio evaluado. Un valor $\mathrm{V}$ mayor a .7 puede considerarse indicador de validez de contenido, valores menores indican la necesidad de reconsiderar el ítem y/o reformularlo.

En la Escala Ciberagredido, los índices generales de Aiken denotan amplio acuerdo entre los jueces, con valores de .72 hasta .80. Es importante destacar que los ítems están bien calificados en cuanto al criterio de pertinencia: los índices de Aiken van de .77 hasta .83 .

En la Tabla 4 se señalan los límites superiores e inferiores para un intervalo de confianza de

Tabla 1

Tipos de ciberagresión sufrida.

Dimensión Agresión Sufrida - Escala Ciberagredido

\begin{tabular}{|c|c|c|}
\hline \multicolumn{3}{|c|}{ Dimensión Agresión Sufrida - Escala Ciberagredido } \\
\hline \multirow{2}{*}{ Forma } & \multicolumn{2}{|c|}{ Explicitación } \\
\hline & Directo & Indirecto \\
\hline \multirow{3}{*}{ Verbal } & $\begin{array}{c}\text { Recibir mensajes escritos ofensivos o } \\
\text { amenazantes a través del teléfono celular o } \\
\text { internet. }\end{array}$ & \\
\hline & & $\begin{array}{l}\text { Una persona envía mensajes escritos ofensivos o } \\
\text { divulga rumores sobre ti entre otras personas a través } \\
\text { del teléfono celular o internet. }\end{array}$ \\
\hline & & $\begin{array}{l}\text { Una persona sube mensajes escritos ofensivos o } \\
\text { amenazantes sobre ti en internet. }\end{array}$ \\
\hline \multirow{3}{*}{ Visual } & $\begin{array}{l}\text { Recibir imágenes ofensivas o amenazantes a } \\
\text { través del celular e internet. }\end{array}$ & \\
\hline & & $\begin{array}{c}\text { Una persona envía fotos o videos ofensivos o } \\
\text { vergonzosos sobre ti a otros, a través del teléfono } \\
\text { celular o internet. }\end{array}$ \\
\hline & & $\begin{array}{c}\text { Una persona sube fotos o videos ofensivos o } \\
\text { vergonzosos sobre ti a través del teléfono celular o } \\
\text { internet. }\end{array}$ \\
\hline Suplantación & & $\begin{array}{c}\text { Una persona usa tu nombre de usuario y contraseña } \\
\text { para hacer cosas ofensivas o amenazantes en tu } \\
\text { nombre a través del celular e internet. }\end{array}$ \\
\hline Exclusión & & $\begin{array}{l}\text { Una persona, sin motivos, no te deja participar en las } \\
\text { redes sociales, juegos o chats online. }\end{array}$ \\
\hline
\end{tabular}


Tabla 2

Tipos de ciberagresión perpetrada.

Dimensión Agresión Perpetrada - Escala Ciberagresor

Forma

Directo

\section{Explicitación}

Enviar mensajes escritos ofensivos o amenazantes a través del teléfono celular o internet.

Verbal

Enviar mensajes escritos ofensivos o divulgar rumores sobre una persona, a través del teléfono celular o internet.

Subir mensajes escritos ofensivos o amenazantes sobre una persona en internet.
Enviar imágenes ofensivas o amenazantes a través del celular e internet.

Visual

Suplantación

Exclusión
Enviar fotos o videos ofensivos o vergonzosos sobre una persona a través del teléfono celular o internet.

Subir fotos o videos ofensivos o vergonzosos sobre una persona a través del teléfono celular o internet.

Usar el nombre de usuario y contraseña de otro para hacer cosas ofensivas o amenazantes en su nombre a través del celular e internet.

Sin motivo, no dejar participar a una persona o personas en las redes sociales, juegos o chats online.

Tabla 3

Valores medios y $\mathrm{V}$ de Aiken de cada ítem.

\begin{tabular}{|c|c|c|c|c|c|c|c|c|}
\hline \multicolumn{9}{|c|}{ ESCALA CIBERAGREDIDO } \\
\hline \multirow[b]{2}{*}{ Ítem } & \multicolumn{2}{|c|}{ Pertinencia } & \multicolumn{2}{|c|}{ Claridad } & \multicolumn{2}{|c|}{ Redacción } & \multicolumn{2}{|c|}{ Índices Generales } \\
\hline & Media & V de Aiken & Media & V de Aiken & Media & V de Aiken & Media & V de Aiken \\
\hline 1 & 4.27 & .82 & 4.13 & .78 & 4.07 & .77 & 4.15 & .79 \\
\hline 2 & 4.33 & .83 & 4.13 & .78 & 4.13 & .78 & 4.20 & .80 \\
\hline 3 & 4.20 & .80 & 3.87 & .72 & 3.80 & .70 & 3.96 & .74 \\
\hline 4 & 4.27 & .82 & 4.00 & .75 & 4.20 & .80 & 4.15 & .79 \\
\hline 5 & 4.13 & .78 & 3.60 & .65 & 3.87 & .72 & 3.87 & .72 \\
\hline 6 & 4.27 & .82 & 3.80 & .70 & 4.07 & .77 & 4.04 & .76 \\
\hline 7 & 4.07 & .77 & 3.93 & .73 & 3.80 & .70 & 3.93 & .73 \\
\hline 8 & 4.20 & .80 & 4.07 & .77 & 4.13 & .78 & 4.13 & .78 \\
\hline 9 & 4.20 & .80 & 4.20 & .80 & 4.13 & .78 & 4.17 & .79 \\
\hline
\end{tabular}

90\%, para los valores obtenidos por cada ítem en cada uno de los criterios evaluados.

Los puntajes inferiores del intervalo de confianza de cada ítem de la escala corroboran el am- plio acuerdo entre los jueces respecto de los tres criterios estudiados, ya que todos son mayores a .50 .

Respecto de la pregunta abierta respondida 
Tabla 4

Valores inferiores y superiores del intervalo de confianza.

\begin{tabular}{|c|c|c|c|c|c|c|c|c|c|c|c|c|c|c|}
\hline \multicolumn{15}{|c|}{ ESCALA CIBERAGREDIDO } \\
\hline \multirow{3}{*}{$\begin{array}{c}\text { Ítem } \\
1\end{array}$} & \multicolumn{4}{|c|}{ Pertinencia } & \multicolumn{4}{|c|}{ Claridad } & \multicolumn{4}{|c|}{ Redacción } & \multicolumn{2}{|c|}{ General } \\
\hline & \multirow{2}{*}{$\frac{\mathbf{M}}{4.27}$} & \multirow{2}{*}{$\frac{\mathbf{V}}{.82}$} & \multicolumn{2}{|c|}{90} & \multirow{2}{*}{$\begin{array}{c}\text { M } \\
4.13\end{array}$} & \multirow{2}{*}{$\begin{array}{c}\mathbf{V} \\
.78\end{array}$} & \multicolumn{2}{|c|}{90} & \multirow{2}{*}{$\frac{\mathbf{M}}{4.07}$} & \multirow{2}{*}{$\begin{array}{c}\mathbf{V} \\
.77\end{array}$} & \multicolumn{2}{|c|}{90} & \multirow{2}{*}{$\begin{array}{c}\mathbf{M} \\
4.15\end{array}$} & \multirow{2}{*}{$\begin{array}{c}\mathbf{V} \\
.79\end{array}$} \\
\hline & & & .72 & .89 & & & .68 & .86 & & & .67 & .84 & & \\
\hline 2 & 4.33 & .83 & .74 & .90 & 4.13 & .78 & .68 & .86 & 4.13 & .78 & .68 & .86 & 4.20 & .80 \\
\hline 3 & 4.20 & .80 & .70 & .87 & 3.87 & .72 & .61 & .80 & 3.80 & .70 & .60 & .79 & 3.96 & .74 \\
\hline 4 & 4.27 & .82 & .72 & .89 & 4.00 & .75 & .65 & .83 & 4.20 & .80 & .70 & .87 & 4.15 & .79 \\
\hline 5 & 4.13 & .78 & .68 & .86 & 3.60 & .65 & .54 & .74 & 3.87 & .72 & .61 & .80 & 3.87 & .72 \\
\hline 6 & 4.27 & .82 & .72 & .89 & 3.80 & .70 & .60 & .79 & 4.07 & .77 & .67 & .84 & 4.04 & .76 \\
\hline 7 & 4.07 & .77 & .67 & .84 & 3.93 & .73 & .63 & .81 & 3.80 & .70 & .60 & .79 & 3.93 & .73 \\
\hline 8 & 4.20 & .80 & .70 & .87 & 4.07 & .77 & .67 & .84 & 4.13 & .78 & .68 & .86 & 4.13 & .78 \\
\hline 9 & 4.20 & .80 & .70 & .87 & 4.20 & .80 & .70 & .87 & 4.13 & .78 & .68 & .86 & 4.17 & .79 \\
\hline
\end{tabular}

por los jueces con sus observaciones acerca de los ítems, se obtuvieron las siguientes sugerencias: dos jueces señalaron que los ítems 3 y 5 podrían indagar por separado las dos conductas que los ítems recogen dentro de la categoría agresión verbal indirecta respectivamente (envío/ publicación de mensajes escritos ofensivos y divulgación de rumores). Dos jueces señalaron la posibilidad de incluir en el ítem 7 otra conducta como es la creación de cuentas falsas en redes sociales. Tras una discusión y análisis con los autores del cuestionario, se acordó que enviar y/o publicar mensajes ofensivos constituye una conducta diferente a divulgar rumores, por lo que se atendió a la sugerencia de los jueces al respecto. El ítem 7 no fue reformulado, dado que la suplantación de identidad constituye una conducta distinta a la asunción de una identidad digital falsa.

\section{Escala Ciberagresor}

La Escala Ciberagresor está conformada por los siguientes ítems:

1. ¿Enviaste mensajes escritos ofensivos o amenazantes a través del teléfono celular o internet?

2. ¿Enviaste imágenes o videos ofensivos o amenazantes a través del teléfono celular o internet?

3. ¿Enviaste mensajes escritos ofensivos o divulgaste rumores sobre alguien a través del teléfono celular o internet?

4. ¿Enviaste imágenes o videos ofensivos o amenazantes sobre alguien a través del teléfono celular o internet?

5. ¿Publicaste mensajes escritos ofensivos o amenazantes, o divulgaste rumores sobre alguien a través del teléfono celular o internet?

6. ¿Publicaste imágenes o videos ofensivos o amenazantes sobre alguien a través del teléfono celular o internet?

7. ¿Utilizaste el nombre de usuario y contraseña de alguien e hiciste cosas ofensivas o amenazantes?

8. ¿Impediste participar, sin motivo, a alguien de alguna actividad en línea (por ej. de un juego o grupo creado en una red social)? 
9. ¿Usaste Internet o el teléfono celular para agredir a otros adolescentes de alguna otra manera? Si la respuesta es sí, describí lo que pasó y marcá la casilla correspondiente.

Las tablas a continuación dan cuenta de las $\mathrm{V}$ de Aiken y los intervalos de confianza al 90\% de los ítems que componen la Escala Ciberagresor. En la Tabla 5 se incluyen los valores medios obtenidos por cada ítem para cada uno de los criterios evaluados $\mathrm{y}$, por último, los puntajes medios obtenidos por cada ítem. Junto a las medias se adjunta el coeficiente $\mathrm{V}$ de Aiken.

En la Escala Ciberagresor se replica la situación hallada en la Escala Ciberagredido: los índices generales de Aiken denotan amplio acuerdo entre los jueces, con valores de .72 hasta .80. Es importante destacar que los ítems están bien calificados en los tres criterios estudiados.

En la Tabla 6 se señalan los límites superiores e inferiores para un intervalo de confianza de 90\%, para los valores obtenidos por cada ítem en cada uno de los criterios evaluados.

Los puntajes inferiores del intervalo de con- fianza de cada ítem de la escala, todos mayores a .50 , corroboran el amplio acuerdo entre los jueces respecto de los tres criterios estudiados.

Respecto de la pregunta abierta respondida por los jueces con sus observaciones acerca de los ítems, se replicaron los mismos comentarios que para la Escala Ciberagredido. Conforme al consenso con los autores del Cuestionario, se tomó la misma decisión respecto de los ítems 3, 5 y 7 de la presente escala.

\section{Discusión}

La vertiginosa incorporación de las tecnologías de la información y la comunicación a la sociedad, y los consecuentes riesgos a los que los adolescentes se enfrentan en internet y las redes sociales ponen en evidencia la urgente necesidad de contar con instrumentos de evaluación que posibiliten el estudio y comprensión de estos fenómenos. El Cuestionario de Ciberagresión de Corcoran y Mc Guckin (2014) es un instrumento específico para medir este fenómeno en este grupo etario. Considerando que la adaptación de instrumentos es valorada como una estrategia más

Tabla 5

Valores medios y V de Aiken de cada ítem.

\begin{tabular}{ccccccccc}
\hline \multicolumn{7}{c}{ ESCALA CIBERAGRESOR } \\
\hline \multicolumn{1}{c}{ Pertinencia } & \multicolumn{2}{c}{ Claridad } & \multicolumn{2}{c}{ Redacción } & \multicolumn{2}{c}{ Índices Generales } \\
Ítem & Media & V de Aiken & Media & V de Aiken & Media & V de Aiken & Media & V de Aiken \\
\hline 1 & 4.13 & .78 & 4.20 & .80 & 4.13 & .78 & 4.15 & .79 \\
2 & 4.20 & .80 & 4.13 & .78 & 4.00 & .75 & 4.11 & .78 \\
3 & 4.20 & .80 & 3.87 & .72 & 3.93 & .73 & 4.00 & .75 \\
4 & 4.13 & .78 & 3.93 & .73 & 4.00 & .75 & 4.02 & .76 \\
5 & 4.20 & .80 & 3.60 & .65 & 3.80 & .70 & 3.87 & .72 \\
6 & 4.07 & .77 & 3.80 & .70 & 4.00 & .75 & 3.96 & .74 \\
7 & 4.27 & .82 & 4.00 & .75 & 4.00 & .75 & 4.09 & .77 \\
8 & 4.27 & .82 & 4.20 & .80 & 4.20 & .80 & 4.22 & .80 \\
9 & 4.33 & .83 & 4.13 & .78 & 4.07 & .77 & 4.17 & .79 \\
\hline
\end{tabular}


Tabla 6

Valores inferiores y superiores del intervalo de confianza.

\begin{tabular}{|c|c|c|c|c|c|c|c|c|c|c|c|c|c|c|}
\hline \multicolumn{15}{|c|}{ ESCALA CIBERAGRESOR } \\
\hline \multirow{3}{*}{$\begin{array}{c}\text { Ítem } \\
1\end{array}$} & \multicolumn{4}{|c|}{ Pertinencia } & \multicolumn{4}{|c|}{ Claridad } & \multicolumn{4}{|c|}{ Redacción } & \multicolumn{2}{|c|}{ General } \\
\hline & \multirow{2}{*}{$\frac{\mathbf{M}}{4.13}$} & \multirow{2}{*}{$\begin{array}{c}\mathbf{V} \\
.78\end{array}$} & \multicolumn{2}{|c|}{90} & \multirow{2}{*}{$\frac{\mathbf{M}}{4.20}$} & \multirow{2}{*}{$\frac{\mathbf{V}}{.80}$} & \multicolumn{2}{|c|}{90} & \multirow{2}{*}{$\frac{\mathbf{M}}{4.13}$} & \multirow{2}{*}{$\begin{array}{c}\mathbf{V} \\
.78\end{array}$} & \multicolumn{2}{|c|}{90} & \multirow{2}{*}{$\frac{\mathbf{M}}{4.15}$} & \multirow{2}{*}{$\begin{array}{c}\mathbf{V} \\
.79\end{array}$} \\
\hline & & & .68 & .86 & & & .70 & .87 & & & .68 & .86 & & \\
\hline 2 & 4.20 & .80 & .70 & .87 & 4.13 & .78 & .68 & .86 & 4.00 & .75 & .65 & .83 & 4.11 & .78 \\
\hline 3 & 4.20 & .80 & .70 & .87 & 3.87 & .72 & .61 & .80 & 3.93 & .73 & .63 & .81 & 4.00 & .75 \\
\hline 4 & 4.13 & .78 & .68 & .86 & 3.93 & .73 & .63 & .81 & 4.00 & .75 & .65 & .83 & 4.02 & .76 \\
\hline 5 & 4.20 & .80 & .70 & .87 & 3.60 & .65 & .54 & .74 & 3.80 & .70 & .60 & .79 & 3.87 & .72 \\
\hline 6 & 4.07 & .77 & .67 & .84 & 3.80 & .70 & .60 & .79 & 4.00 & .75 & .65 & .83 & 3.96 & .74 \\
\hline 7 & 4.27 & .82 & .72 & .89 & 4.00 & .75 & .65 & .83 & 4.00 & .75 & .65 & .83 & 4.09 & .77 \\
\hline 8 & 4.27 & .82 & .72 & .89 & 4.20 & .80 & .70 & .87 & 4.20 & .80 & .70 & .87 & 4.22 & .80 \\
\hline 9 & 4.33 & .83 & .74 & .90 & 4.13 & .78 & .68 & .86 & 4.07 & .77 & .67 & .84 & 4.17 & .79 \\
\hline
\end{tabular}

rápida, práctica y económica que la construcción de cuestionarios y test nuevos (Cardoso-Ribeiro, Gómez-Conesa, \& Hidalgo-Montesinos, 2010) $\mathrm{y}$, ante la vacancia de instrumentos con esta característica y la fiabilidad demostrada por este en población irlandesa, se considera valiosa su adaptación a la lengua y cultura argentina. Tras un riguroso proceso de adaptación transcultural se arribó a la versión argentina preliminar del cuestionario. El juicio de expertos permitió concluir la fase de adaptación de un instrumento creado para otra población, a la nuestra, garantizando así su equivalencia cultural. Su validación en términos de contenido posibilitó la determinación de indicadores rigurosos de la representatividad de cada uno de los ítems de cada escala.

Uno de los aportes de la fase de adaptación del cuestionario a nuestro contexto ha sido la denominación de las escalas que lo componen: Escala Ciberagredido y Escala Ciberagresor. La expresión Ciberagredido evita confundir a la ciberagresión sufrida con cibervictimización. El respeto por el carácter específico del constructo ciberagresión, evitando su solapamiento con el cyberbullying, torna valiosa esta contribución.

En el juicio de expertos, cada juez realizó una valoración cuantitativa y cualitativa de cada ítem del cuestionario, atendiendo a tres categorías: pertinencia, claridad y redacción. Los resultados cuantitativos se sistematizaron utilizando el coeficiente de validez $\mathrm{V}$ de Aiken complementado con el uso de intervalos de confianza. En la Escala Ciberagredido, los índices generales de Aiken denotan amplio acuerdo entre los jueces, con valores que varían desde .72 hasta .80 , evidenciando el alto grado de consenso de los expertos consultados respecto de la representatividad y adecuación lingüística de cada ítem. Asimismo, los puntajes inferiores del intervalo de confianza de cada ítem ratifican la alta conformidad entre los jueces respecto de los tres criterios estudiados ya que todos son mayores a .50. En la Escala Ciberagresor se replica la situación descripta para la Escala Ciberagredido. Estos resultados permiten concluir que los ítems que componen ambas escalas del Cuestionario de Ciberagresión presentan evidencias contundentes acerca de la validez de contenido de este.

Respecto de los resultados cualitativos, cabe destacar que las aportaciones realizadas por los jueces fueron cuidadosamente discutidas y analizadas por los investigadores. En el caso de los 
ítems 3 y 5 de ambas escalas, los jueces aportaron que se podrían indagar por separado las dos conductas que estos recogen dentro de la categoría agresión verbal indirecta (envío/ publicación de mensajes escritos ofensivos y divulgación de rumores respectivamente). Del análisis practicado, se concluye que enviar y/o publicar mensajes ofensivos constituye una conducta diferente a divulgar rumores dado que esta última implica la difamación a través de la divulgación de información que puede no ser fidedigna. Si bien ambas conductas persiguen como objetivo dañar la posición social del ciberagredido a través del uso de textos escritos que se dirigen o comparten con terceros, se cristalizan mediante acciones cualitativamente distintas. Se resolvió atender a la sugerencia de los jueces dado que esta respeta y enriquece la tipología de ciberagresión verbal indirecta que se utiliza en el cuestionario. En el caso del ítem 7 de ambas escalas, los jueces señalaron la posibilidad de incluir en esta otra conducta como es la creación de cuentas falsas en redes sociales. Sin embargo, en este caso, los investigadores decidieron no reformular este ítem, por concluir que esta conducta no se ajusta a la definición de esta tipología, considerando que la suplantación de identidad se refiere a situaciones en las que alguien se hace pasar por el ciberagredido a través del teléfono móvil o internet, para burlarse de él o causarle problemas (Nocentini et al., 2010).

Se reconoce como una de las limitaciones del presente estudio la ausencia de validación de contenido del cuestionario en su contexto original de producción; sin embargo, consideramos a esta investigación como un valioso aporte dado que ofrece el primer instrumento en español válido para medir la ciberagresión en adolescentes escolarizados. Lacunza et al. (2019) señalan, en su estudio bibliométrico en torno al comportamiento agresivo de adolescentes latinoamericanos me- diado por la virtualidad, la vacancia de investigaciones sobre este tópico en nuestro país, así como la ausencia de instrumentos de evaluación validados a nivel local. Este estudio, al aportar un instrumento validado científicamente en nuestra población, resuelve algunas de dichas vacancias. La investigación continuará, en colaboración con los autores del cuestionario, con el análisis de las propiedades psicométricas del cuestionario. Este hecho posibilitará no solo el estudio del fenómeno en nuestro país sino también la realización de futuras investigaciones comparativas.

Uno de los resultados esperados es contribuir con la realización de estudios empíricos que arrojen mayor claridad sobre los vínculos entre los jóvenes y la tecnología, posibilitando el diseño de políticas educativas en torno a ciudadanía y alfabetización digital.

\section{Referencias}

Aiken, L. (1980). Content validity and reliability of single items or questionnaires. Educational and Psychological Measurement, 40(4), 955-959. doi: 10.1177/001316448004000419

Álvarez-García, D., Barreiro-Collazo, A., \& Núñez, J. C. (2017). Cyberaggression among adolescents: Prevalence and gender differences. Comunicar, 25(50), 8997. doi: 10.3916/c50-2017-08

Álvarez-García, D., Dobarro, A., \& Núñez, J. C. (2015). Validez y fiabilidad del Cuestionario de Cibervictimización en Estudiantes de Secundaria. Aula Abierta, 43(1), 32-38. doi: 10.1016/j.aula.2014.11.001

Bauman, S., Underwood, M., \& Card, N. (2013). Definitions: Another perspective and a proposal for a beginning with cyberagression. En S. Bauman, D. Cross \& J. Walker (Eds.), Principles of Cyberbullying Research: Definitions, Measures, and Methodology (pp. 41-46). Londres: Routledge. ISBN: 978-0415-89749-5 
Best, S., Ré, N., Mc Guckin, C., Corcoran, L., \& Casasnovas, A. (2017). Retos y desafíos de la adaptación transcultural del Cuestionario de Ciberagresión en una muestra de estudiantes argentinos. Subjetividad y Procesos Cognitivos, 21(2), 17-41. ISSN: 1852-7310. Recuperado de http:/www.scielo.org.ar/scielo.php?scrip$\mathrm{t}=$ sci_arttext\&pid=S1852-73102017000200001\&ln$\mathrm{g}=\mathrm{es} \& \mathrm{t} \operatorname{lng}=\mathrm{es}$

Brighi, A., Ortega, R., Scheitauer, H., Smith, P. K., Tsormpatzoudis, C., Barkoukis, V., ... \& Thompson, J. (2012). European Bullying Intervention Project Questionnaire (ECIPQ). University of Bologna. Unpublished manuscript.

Bringué-Sala, X., Sádaba-Chalezquer, C., \& Artopoulos, A. (2014). La generación interactiva en Argentina: Niños y adolescentes ante las pantallas. Education Policy Analysis Archives/Archivos Analíticos de Políticas Educativas, 22,1-19. Recuperado de https:// www.redalyc.org/pdf/2750/275031898066.pdf

Bulger, S. M., \& Housner, L. D. (2007). Modified delphi investigation of exercise science in physical education teacher education. Journal of Teaching in Physical Education, 26(1), 57-80. doi: 10.1123/jtpe.26.1.57

Cabero-Almenara, J., \& Llorente-Cejudo, M. del C. (2013). La aplicación del juicio de experto como técnica de evaluación de las tecnologías de la información (TIC). Eduweb: Revista de Tecnología de Información y Comunicación en Educación, 7(2), 11-22. Recuperado de http://servicio.bc.uc.edu.ve/educacion/ eduweb/v7n2/art01.pdf

Cardoso-Ribeiro, C., Gómez-Conesa, A., \& Hidalgo-Montesinos, M. D. (2010). Metodología para la adaptación de instrumentos de evaluación. Revista Fisioterapia, 32(6), 264-270. doi: 10.1016/j.ft.2010.05.001

Cicchetti, D. V. (1994). Guidelines, criteria, and rules of thumb for evaluating normed and standardized assessment instruments in psychology. Psychological Assessment, 6(4), 284-290. doi: 10.1037/10403590.6.4.284

Corcoran, L. (2013). Traditional Bullying and Cyberbullying at Post-Primary School Level in Ireland: Coun- tering the Aggression and Buffering its Negative Psychological Effects. (Tesis doctoral). Recuperado de http:/www.tara.tcd.ie/handle/2262/85396

Corcoran, L., \& Mc Guckin, C. (2014). The incidence of bullying and aggression in Irish post-primary schools: An investigation of school and cyber settings. In Proceedings of Annual Conference of the Educational Studies Association of Ireland, Sheraton Hotel, Athlone, Ireland, 10-12 April 2014.

Corcoran, L., Mc Guckin, C., \& Prentice, G. (2015). Cyberbullying or cyber aggression? A review of existing definitions of cyber-based peer-to-peer aggression. Societies, 5(2), 245-255. doi: 10.3390/soc5020245

Escobar-Pérez, J., \& Cuervo-Martínez, Á. (2008). Validez de contenido y juicio de expertos: Una aproximación a su utilización. Avances en Medición, 6, 2736. Recuperado de http://www.humanas.unal.edu. co/lab_psicometria/revista-avances-en-medicion/ avances-en-medicion-no6

Escurra-Mayaute, L. M. (1988). Cuantificación de la Validez de Contenido por Criterio de Jueces. Revista de Psicología - PUCP, 6(1-2), 103-111. Recuperado de http://revistas.pucp.edu.pe/index.php/psicologia/ article/view/4555

Fidler, F. (2002). The fifth edition of the APA Publication Manual: Why its statistics recommendations are so controversial. Educational and Psychological Measurement, 62(5), 749-770. doi: 10.1177/001316402236876

Gámez-Guadix, M., Villa-George, F., \& Calvete, E. (2014). Psychometric properties of the Cyberbullying Questionnaire (CBQ) among Mexican adolescents. Violence and Victims, 29(2), 232-247. doi: 10.1891/08866708.vv-d-12-00163r1

Garaigordobil, M. (2011). Prevalencia y consecuencias del cyberbullying: Una revisión. International Journal of Psychology and Psychological Therapy, 11(2) 233-254. Recuperado de https://www.ijpsy.com/volumen11/num2/295.html

Garaigordobil, M. (2013). Cyberbullying. Screening de acoso entre iguales. Madrid: TEA. 
Grigg, D. W. (2010). Cyber-Aggression: Definition and concept of cyberbullying. Australian Journal of Guidance and Counselling 20(2), 143-156. doi: 10.1375/ ajgc.20.2.143

Herrera-López, M., Casas, J. A., Romera, E. M., Ortega-Ruiz, R., \& del Rey, R. (2017). Validation of the European Cyberbullying Intervention Project Questionnaire for Colombian adolescents. Cyberpsychology, Behavior, and Social Networking, 20(2), 117125. doi: 10.1089/cyber.2016.0414

Hinduja, S., \& Patchin, J. W. (2015). Bullying Beyond the Schoolyard (2a ed.). Thousand Oaks, CA: Corwin.

Hunter, S. C., Boyle, J. M. E., \& Warden, D. (2004). Help seeking amongst child and adolescent victims of peer-aggression and bullying: The influence of schoolstage, gender, victimisation, appraisal, and emotion. British Journal of Educational Psychology, 74(3), 375-390. doi: 10.1348/0007099041552378

Jiménez, A. E., Castillo, V. D., \& Cisternas, L. C. (2012). Validación de la escala de agresión entre pares, y subescala de agresión virtual en escolares chilenos. Revista Latinoamericana de Ciencias Sociales Niñez y Juventud, 10(2), 825-840. Recuperado de http://revistaumanizales.cinde.org.co

Kowalski, R. M., Giumetti, G. W., Schroeder, A. N., \& Lattanner, M. R. (2014). Bullying in the digital age: A critical review and meta-analysis of cyberbullying research among youth. Psychological Bulletin, 140(4), 1073-1137. doi: 10.1037/a0035618

Laca-Arocena, F. A. V., Pérez-Verduzco, G., Luna-Bernal, A. C. A., Carrillo-Ramírez, E., \& Garaigordobil, M. (2020). Propiedades psicométricas del Test Cyberbullying en una muestra de adolescentes mexicanos estudiantes de bachillerato. Revista Evaluar, 20(2), 1-19. Recuperado de https://revistas.unc.edu.ar/index.php/revaluar

Lacunza, A. B., Contini, E. N., Caballero, S. V., \& Mejail, S. M. (2019). Agresión en las redes y adolescencia: Estado actual en América Latina desde una perspectiva bibliométrica. Investigación y Desarrollo, 27(2), 6-32. Recuperado de https://rcientificas.uninorte. edu.co

Langos, C. (2012). Cyberbullying: The challenge to define. Cyberpsychology, Behavior, and Social Networking, 15(6), 285-289. doi: 10.1089/cyber.2011.0588

Machmutow, K., Perren, S., Sticca, F., \& Alsaker, F. D. (2012). Peer victimisation and depressive symptoms: Can specific coping strategies buffer the negative impact of cybervictimisation? Emotional and Behavioural Difficulties, 17(3-4), 403-420. doi: 10.1080/13632752.2012.704310

McGartland-Rubio, D., Berg-Weger, M., Tebb, S. S., Lee, E. S., \& Rauch, S. (2003). Objectifying content validity: Conducting a content validity study in social work research. Social Work Research, 27(2), 94-104. doi: 10.1093/swr/27.2.94

Menesini, E., Nocentini, A., \& Calussi, P. (2011). The measurement of cyberbullying: Dimensional structure and relative item severity and discrimination. $C y$ berpsychology, Behavior, and Social Networking, 14(5), 267-274. doi: 10.1089/cyber.2010.0002

Merino-Soto, C. (2018). Confidence interval for difference between coefficients of content validity (Aiken's V): A SPSS syntax. Anales de Psicología, 34(3), 587590. doi: 10.6018/analesps.34.3.283481

Merino-Soto, C., \& Livia-Segovia, J. (2009). Intervalos de confianza asimétricos para el índice la validez de contenido: Un programa Visual Basic para la $\mathrm{V}$ de Aiken. Anales de Psicología, 25(1), 169-171. Recuperado de https://revistas.um.es/analesps

Montero, I., \& León, O. (2002). Clasificación y descripción de las metodologías de investigación en Psicología. International Journal of Clinical and Health Psychology, 2(3), 503-508. Recuperado de http://www. aepc.es/ijchp

Muñiz, J., \& Hambleton, R. K. (1996). Directrices para la traducción y adaptación de los tests. Papeles del Psicólogo, 66, 63-70. Recuperado de http://www.papelesdelpsicologo.es

Nocentini, A., Calmaestra, J., Schultze-Krumbholz, A., Scheithauer, H., Ortega, R., \& Menesini, E. (2010). Cyberbullying: Labels, behaviours and definition 
in three European countries. Australian Journal of Guidance and Counselling, 20(2), 129-142. doi: 10.1375/ajgc.20.2.129

Olweus, D. (2010). Understanding and researching bullying: Some critical issues. En S. R. Jimerson, S. M. Swearer \& D. L. Espelage (Eds.), Handbook of Bullying in Schools: An International Perspective (pp. 9-33). UK: Routledge/Taylor \& Francis.

Olweus, D. (2012). Cyberbullying: An overrated phenomenon? European Journal of Developmental Psychology, 9(5), 520-538. doi: 10.1080/17405629.2012.682358

Paolini, P., \& Ravalli, M. J. (2016). Kids online. Chic@s conectados: Investigación sobre percepciones y hábitos de niños, niñas y adolescentes en internet $y$ redes sociales. Buenos Aires, Argentina: Fondo de las Naciones Unidas para la Infancia. Recuperado de https://www.unicef.org/argentina/informes/kids-online-chics-conectados

Paul, S., Smith, P. K., \& Blumberg, H. H. (2012). Investigating legal aspects of cyberbullying. Psicothema, 24(4), 640-645. Recuperado de http://www.psicothema.com

Penfield, R. D., \& Giacobbi, P. R. (2004). Applying a score confidence interval to Aiken's item content-relevance index. Measurement in Physical Education and Exercise Science, 8(4), 213-225. doi: 10.1207/ s15327841mpee0804_3

Secretaría de Evaluación Educativa. (2017). Aprender 2017: Informe de resultados - Secundaria. Buenos Aires, Argentina: Ministerio de Educación. Recuperado de https://www.argentina.gob.ar/noticias/aprender-2017-accede-los-resultados-de-primaria-y-secundaria-nivel-nacional

Skjong, R., \& Wentworth, B. H. (2001). Expert judgement and risk perception. The Eleventh International Offshore and Polar Engineering Conference. Recuperado de http://research.dnv.com/skj/Papers/SkjWen. pdf

Smith, P. K., \& Berkkun, F. (2017). How research on cyberbullying has developed. En C. Mc Guckin \& L. Corcoran (Eds.). Bullying and cyberbullying: Prevalen- ce, psychological impacts and intervention strategies (pp. 11-17). Hauppauge, NY: Nova Science.

Sticca, F., Machmutow, K., Stauber, A., Perren, S., Palladino, B., Nocentini, A., ... \& Guckin, C. (2015). The Coping with Cyberbullying Questionnaire: Development of a new measure. Societies, 5(2), 515-536. doi: $10.3390 /$ soc5020515

Yahner, J., Dank, M., Zweig, J. M., \& Lachman, P. (2015). The co-occurrence of physical and cyber dating violence and bullying among teens. Journal of Interpersonal Violence, 30(7), 1079-1089. doi: 10.1177/0886260514540324

Zych, I., Ortega-Ruiz, R., \& Marín-López, I. (2016). Cyberbullying: A systematic review of research, its prevalence and assessment issues in Spanish studies. Revista Psicología Educativa, 22(1), 5-18. doi: 10.1016/j.pse.2016.03.002

Zych, I., Ortega-Ruiz, R., \& del Rey, R. (2015). Scientific research on bullying and cyberbullying: Where have we been and where are we going. Aggression and Violent Behavior, 24, 188-198. doi: 10.1016/j. avb.2015.05.015 